\title{
Correlation of Obesity with External Cephalic Version Success among Women with One Previous Cesarean Delivery
}

\author{
Rodney McLaren Jr., MD ${ }^{1} \quad$ Fouad Atallah, MD ${ }^{1} \quad$ Nelli Fisher, MD ${ }^{1} \quad$ Howard Minkoff, MD ${ }^{1}$ \\ ${ }^{1}$ Department of Obstetrics and Gynecology, Maimonides Medical \\ Center, Brooklyn, New York \\ Address for correspondence Rodney A. McLaren, Jr., MD, Department \\ of Obstetrics and Gynecology, Maimonides Medical Center, 967 48th \\ Am J Perinatol Rep 2020;10:e324-e329. \\ Street, Brooklyn, NY 11201 (e-mail: rmclaren624@gmail.com).
}

\begin{abstract}
Objective Our aim was to assess the correlation of body mass index (BMI) with the success rate of external cephalic version (ECV) among women with one prior cesarean delivery.

Study Design A cross-sectional study of pregnant women with one previous cesarean delivery who underwent ECV. The relationship between BMI and success rate of ECV was assessed. Adverse outcomes were also compared between women with an ECV attempt, and women who had a repeat cesarean delivery. Data were extracted from the U.S. Natality Database from 2014 to 2017. Pearson's correlation coefficient was performed to assess the relationship between BMI and success rate of ECV.

Results There were 2,329 women with prior cesarean delivery underwent an ECV

Keywords

- external cephalic version

- prior cesarean delivery

- obesity

- vaginal birth after cesarean attempt. The success rate of ECV among the entire cohort was $68.3 \%$. There was no correlation between BMI and success rate of ECV $(r=0.024, p=0.239)$. Risks of adverse maternal and neonatal outcomes were similar between the ECV attempt group and the repeat cesarean delivery group.

Conclusion There was no correlation of BMI with the rate of successful ECV among women with one prior cesarean delivery. Given the similar success rates of ECV and adverse outcomes, obese women with one prior cesarean delivery should be offered ECV.
\end{abstract}

The cesarean delivery rate in the United States has hovered around $32 \%,{ }^{1}$ which is higher than many experts suggest would be optimal. ${ }^{2,3}$ Guidelines have been written and efforts have been made to lower the cesarean delivery rate. ${ }^{4,5}$ One way to lower the rate is by increasing the number of trials of labor after cesarean delivery among patients that are eligible. Trial of labor after cesarean delivery and vaginal birth after cesarean delivery has been shown to be safe. ${ }^{6}$ However, it has been reported that only approximately $25 \%$ of women undergo trial of labor after cesarean delivery, ${ }^{7}$ and that the current rate of vaginal birth after cesarean delivery is $13.3 \%{ }^{1,8}$

received

March 6, 2020

accepted

May 13, 2020
DOI https://doi.org/

10.1055/s-0040-1715173. ISSN 2157-6998.
One of the indications for repeat cesarean delivery is breech presentation. ${ }^{9}$ Accordingly, external cephalic version (ECV) could help reduce the overall cesarean delivery rate. ${ }^{10,11} \mathrm{We}$, and others, have previously shown that ECV is generally safe among women with a prior cesarean delivery. ${ }^{12-21}$ Since ECV involves manual manipulation of the fetus through the maternal abdomen, obesity may affect the efficacy of ECV. This is particularly noteworthy since the rate and degree of obesity in reproductive age women in the United States has been increasing, with the latest reported prevalence being over 30\%. ${ }^{22,23}$ One prior study demonstrated a decreased success rate of ECV as well as a decreased vaginal birth after cesarean delivery rate

Copyright @ 2020 by Thieme Medical Publishers, Inc., 333 Seventh Avenue, New York, NY 10001, USA. Tel: +1(212) 760-0888.
License terms

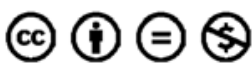


among women with class III obesity compared with normal weight. $^{24}$ Whether this effect of obesity is also seen among women with one prior cesarean delivery is unknown. The objective of this study was to evaluate the correlation of obesity with the success rate of ECV among women with one prior cesarean delivery.

\section{Study Design}

We performed a cross-sectional study among women with one prior cesarean delivery who underwent an external cephalic version (ECV). Data were extracted from the U.S. natality database, which contains data generated from birth certificates, from 2014 to 2017 . This is a publicly available dataset that contains deidentified data, and thus, institutional review board approval was not required.

Analysis was limited to singleton, nonanomalous gestations among women with one prior cesarean delivery who had an attempted ECV. We excluded pregnancies without a documented body mass index (BMI), and those that delivered before 36 completed weeks. Given that women with a breech and a prior cesarean delivery are offered either an ECV or a repeat cesarean delivery, we also included women who had a repeat cesarean delivery with fetal breech presentation without an attempted ECV when we evaluated the secondary outcomes listed below.

The primary outcome was successful ECV. Secondary outcomes included mode of delivery, and adverse maternal and neonatal outcomes. Adverse maternal outcomes included uterine rupture, maternal transfusion, unplanned hysterectomy, and intensive care unit admission. Adverse neonatal outcomes included 5-minute Apgar's score less than 7, immediate assisted ventilation, assisted ventilation needed for more than 6 hours, and neonatal intensive care unit admission. Adverse maternal and neonatal outcomes were compared between those women with a fetal breech presentation who had an attempted ECV and those who had a repeat cesarean delivery.

Maternal and labor characteristics were extracted from the dataset. Maternal characteristics included maternal age, BMI at time of delivery admission, weight gain, history of prior vaginal deliveries, gestational diabetes, pregestational diabetes, gestational hypertension, chronic hypertension during pregnancy while labor characteristics included gestational age at delivery, mode of delivery, and birthweight.

Pearson's correlation coefficient was performed to determine the relationship between BMI and the success rate of ECV. Among women with a successful ECV and a trial of labor, the relationship between maternal obesity and rate of vaginal birth after cesarean delivery was also assessed using Pearson's correlation. For secondary analyses, continuous variables were compared using Student's t-test and categorical variables were compared using Chi-square test. Multivariable logistic regression analysis was performed to predict adverse maternal and neonatal outcomes among women with attempted ECV and women with fetal breech, repeat cesarean delivery controlling for potential confounders including maternal age, gestational age, gestational weight gain, gestational diabetes, pregestational diabetes, gestational hypertension, chronic hyperten- sion, and birthweight. StataCorp LLC Stata 15.1 (College Station, TX) was used to perform all analyses.

\section{Results}

There was a total of $1,631,025$ (10.3\%) women with one prior cesarean delivery among $15,807,774$ births that occurred between 2014 and 2017. After exclusions (1,460,953 women either without an ECV attempt or it was unknown whether there had been an attempt, 98,200 women with gestational age of less than 36 weeks, 61,752 women with multigestations, 7,695 neonates with congenital anomalies, and 96 women who had no reported BMI or trial of labor), there was a total of 2,329 women available for analyses (-Fig. 1).

There was a total of 1,590 women who had a successful ECV for a success rate of $68.3 \%$ among the entire cohort. There was a poor correlation between BMI and the success rate of $\operatorname{ECV}(r=0.024, p=0.239)$. Among women with a successful ECV, 778 women had a trial of labor after cesarean delivery with an overall vaginal birth after cesarean delivery rate of $74.6 \%$. There was a poor correlation between BMI and vaginal birth after cesarean delivery rate among women with a successful ECV $(r=-0.064, p=0.076)$.

Maternal and neonatal characteristics of women with an ECV attempt and women with a repeat cesarean delivery without labor are shown in - Table 1. Women with an ECV attempt were younger, had a lower BMI, and delivered at a later gestational age with a larger neonate birthweight.

Secondary outcomes are shown on - Tables 2 and 3. Obese women with breech fetuses who had an attempted ECV had similar risks of adverse maternal and neonatal outcomes compared with obese women who had a repeat cesarean delivery without a trial of labor, a finding also seen among women with BMI less than $30 \mathrm{~kg} / \mathrm{m}^{2}$. There were two uterine ruptures in the entire cohort, one woman with BMI of 16.8 $\mathrm{kg} / \mathrm{m}^{2}$ and the other one with BMI of $25.5 \mathrm{~kg} / \mathrm{m}^{2}$, both after successful ECV, with one diagnosed after failed trial of labor after cesarean delivery and the other without a trial of labor.

\section{Discussion}

We found that maternal obesity does not have an effect on the success rate of ECV among women with one prior cesarean delivery. The previous literature on the effect of obesity on external cephalic version among women with no prior cesarean deliveries has been conflicting.

Several studies have suggested that higher BMI portends lower ECV success rates. In one small prospective study, women with a BMI greater than $25 \mathrm{~kg} / \mathrm{m}^{2}$ were reported to have a lower success rate of ECV compared with women with a BMI less than $25 \mathrm{~kg} / \mathrm{m}^{2}{ }^{25}$ Another study found that a BMI less than $25 \mathrm{~kg} / \mathrm{m}^{2}$ was associated with successful ECV among 603 women. ${ }^{26}$ Mauldin et al also found that a higher maternal weight was negatively associated with successful ECV among 203 ECV attempts. ${ }^{27}$ Finally, in the largest study $(n=51,002)$ evaluating the effect of BMI on ECV success rates, Chaudhary et al found that the success rates of ECV decreased as the BMI increased (going from 65 to 58.5\%). ${ }^{24}$ 


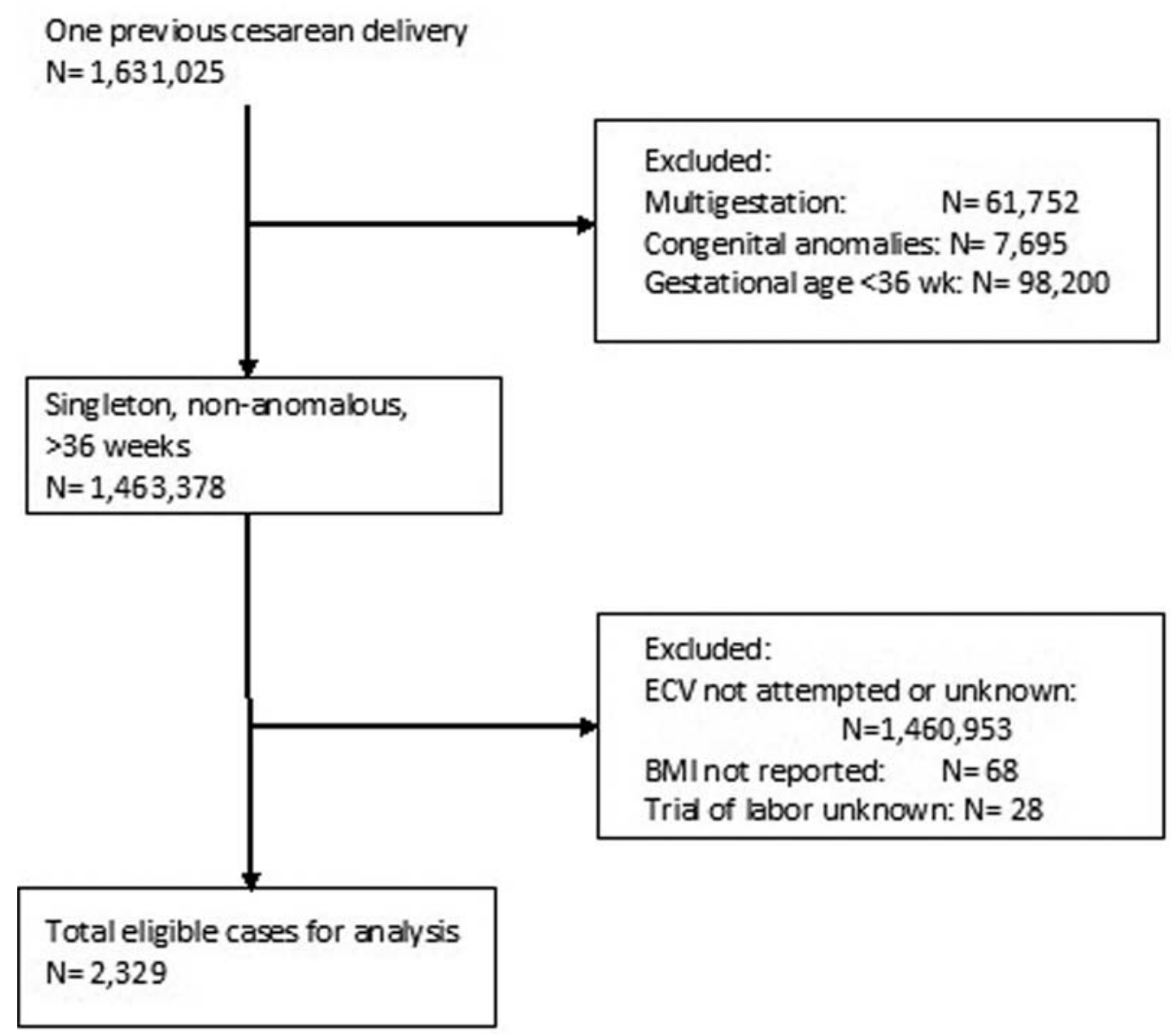

Fig. 1 Cohort selection flow diagram.

Table 1 Maternal characteristics and birthweight among external cephalic version attempts and repeat cesarean deliveries

\begin{tabular}{|l|l|l|l|}
\hline & $\begin{array}{l}\text { ECV attempted } \\
(\boldsymbol{n}=\mathbf{2 , 3 2 9 )}\end{array}$ & $\begin{array}{l}\text { Repeat cesarean delivery } \\
(\boldsymbol{n}=\mathbf{4 2 , 5 3 9 )}\end{array}$ & $\boldsymbol{p}$-Value \\
\hline Maternal age & $30.9 \pm 5.5[1,931]$ & $31.6 \pm 5.4$ & $<0.001$ \\
\hline Body mass index & $27.9 \pm 7.0$ & $28.6 \pm 7.9$ & $<0.001$ \\
\hline Gestational weight gain & $29.1 \pm 14.5[1,912]$ & $29.2 \pm 15.1[42,095]$ & 0.674 \\
\hline History of pregestational diabetes & $20(1.0)[1,931]$ & $928(2.2)$ & 0.001 \\
\hline History of gestational diabetes & $187(9.7)[1,931]$ & $4,157(9.8)$ & 0.899 \\
\hline History of chronic hypertension & $45(2.3)[1,931]$ & $1,393(3.3)$ & 0.022 \\
\hline History of gestational hypertension & $85(4.4)[1,931]$ & $2,516(5.9)$ & 0.006 \\
\hline Gestational age & $39.0 \pm 1.5[1,930]$ & $38.7 \pm 1.5[42,524]$ & $<0.001$ \\
\hline Birthweight & $3,427.1 \pm 502.3[1,930]$ & $3,346.8 \pm 540.6[42,531]$ & $<0.001$ \\
\hline
\end{tabular}

Abbreviation: ECV, external cephalic version.

Data are mean, \pm standard deviation, [ $n$, if missing data].

In contrast, others have found no effect of obesity on the success rate of ECV. Hellström et al performed a multivariable analysis of 300 women who underwent $\mathrm{ECV}$, and reported that obesity was not one of the significant variables for success prediction. ${ }^{28}$ A similar study also found that only amniotic fluid and fetal weight out of six variables, including obesity, was predictive of a successful ECV. ${ }^{29}$ In addition, in an analysis looking at 10 factors among 108 ECV attempts, maternal weight was not associated with successful ECV. ${ }^{30}$ Finally, in a randomized controlled trial evaluating the use of tocolytics during ECV attempt, maternal weight was found to have no effect on success of ECV. ${ }^{31}$ Our current study, limited to women with a prior cesarean delivery, found no effect of obesity on ECV success rates among a larger sample size of women. 
Table 2 Adverse outcomes after external cephalic version attempt and repeat cesarean delivery among nonobese women

\begin{tabular}{|c|c|c|c|}
\hline & $\begin{array}{l}\text { ECV attempt } \\
(n=1,587)\end{array}$ & $\begin{array}{l}\text { Repeat cesarean delivery } \\
(n=27,479)\end{array}$ & ${ }^{\mathrm{a} A d j u s t e d ~ O R}$ \\
\hline \multicolumn{4}{|l|}{ Adverse maternal outcomes } \\
\hline Blood transfusion & $11(0.8)[1,300]$ & $130(0.5)[27,451]$ & $1.85[1.00-3.45]$ \\
\hline Uterine rupture & $2(0.2)[1,300]$ & $16(0.06)[27,451]$ & $2.67[0.61-11.73$ \\
\hline Unplanned hysterectomy & $1(0.08)[1,300]$ & $37(0.1)[27,451]$ & $0.77[0.11-5.66]$ \\
\hline Admission to ICU & $4(0.3)[1,300]$ & $63(0.2)[27,451]$ & $1.63[0.59-4.50]$ \\
\hline \multicolumn{4}{|l|}{ Adverse neonatal outcomes } \\
\hline 5-minute Apgar's score $<7$ & $25(1.9)[1,300]$ & $427(1.6)[27,479]$ & $1.46[0.97-2.20]$ \\
\hline Immediate assisted ventilation & $41(3.2)[1,300]$ & $1,203(4.4)[27,462]$ & $0.79[0.58-1.09]$ \\
\hline Assisted ventilation for $>6$ hours & $9(0.7)[1,300]$ & $274(1.0)[27,462]$ & $0.85[0.44-1.66]$ \\
\hline Admission to NICU & 73 (5.6) $[1,300]$ & 1,993 (7.3) $[27,462]$ & $0.92[0.72-1.18]$ \\
\hline
\end{tabular}

Abbreviations: ECV, external cephalic version; NICU, neonatal intensive care unit; OR, odds ratio.

Data are $n(\%)$ and $[n$, if missing data].

a Outcomes adjusted for maternal age, gestational weight gain, gestational age, gestational diabetes, pregestational diabetes, gestational hypertension, chronic hypertension, and birthweight.

Table 3 Adverse outcomes after external cephalic version attempt and repeat cesarean delivery among obese women

\begin{tabular}{|c|c|c|c|}
\hline & $\begin{array}{l}\text { ECV attempt } \\
(n=742)\end{array}$ & $\begin{array}{l}\text { Repeat cesarean delivery } \\
(n=15,060)\end{array}$ & adjusted OR \\
\hline \multicolumn{4}{|l|}{ Adverse maternal outcomes } \\
\hline Blood transfusion & $3(0.5)[631]$ & $95(0.6)[15,045]$ & $0.53[0.13-2.14]$ \\
\hline Uterine rupture & 0 & $8(0.05)[15,045]$ & \\
\hline Unplanned hysterectomy & $3(0.5)[631]$ & $28(0.2)[15,045]$ & $2.04[0.48-8.66]$ \\
\hline Admission to ICU & $2(0.3)[631]$ & $45(0.3)[15,045]$ & $1.20[0.29-5.00]$ \\
\hline \multicolumn{4}{|l|}{ Adverse neonatal outcomes } \\
\hline 5-minute Apgar's score $<7$ & $11(1.7)$ [631] & $384(2.5)$ & $0.78[0.43-1.44]$ \\
\hline Immediate assisted ventilation & $31(4.9)[631]$ & $1,107(7.4)[15,051]$ & $0.73[0.51-1.06]$ \\
\hline Assisted ventilation for $>6$ hours & $4(0.6)[631]$ & $275(1.8)[15,051]$ & $0.42[0.16-1.13]$ \\
\hline Admission to NICU & $40(6.3)[631]$ & $1,685(11.2)[15,051]$ & $0.65[0.47-0.90]$ \\
\hline
\end{tabular}

Abbreviations: ECV, external cephalic version; NICU, neonatal intensive care unit; OR, odds ratio.

Data are $n(\%)$ and $[n$, if missing data].

a Outcomes adjusted for maternal age, gestational weight gain, gestational age, gestational diabetes, pregestational diabetes, gestational hypertension, chronic hypertension, and birthweight.

Although several studies have focused on the safety of ECV among women with previous cesarean delivery compared with women without prior cesarean delivery, little has been reported on the effect of obesity on success rate of ECV in that population. When evaluating for confounders between women with $(n=38)$ and without a previous cesarean $(n=62)$, one author found no effect of obesity on the success of ECV. ${ }^{20}$ We report a similar finding in a larger sample.

We also found that there was no increased risk of adverse maternal or neonatal outcomes when there was an ECV attempt compared with when a repeat cesarean for breech presentation was performed. This held among both nonobese and obese women. The effect of obesity on trials of labor after cesarean deliveries has been studied. A large retrospective cohort study of 538,264 obese pregnancies found that women who underwent trial of labor after cesarean delivery had an increased risk of adverse maternal and neonatal outcomes. ${ }^{32}$ The different results seen in that study, contrasted with ours, may be due to the inclusion of women with two prior cesarean deliveries in their cohort. Another retrospective cohort study of 344 women with BMI greater than $50 \mathrm{~kg} / \mathrm{m}^{2}$ found that labor, compared with planned cesarean delivery, was associated with lower rates of maternal and neonatal morbidity. ${ }^{33}$ However, Hibbard et al found, in a secondary analysis of a large prospective trial of women undergoing trial of labor after cesarean delivery, that increasing obesity was associated with increased risk of trial failure and maternal and neonatal morbidity. ${ }^{34}$ In contrast, we found that increasing BMI had no effect on the risk of failed trial of labor and adverse maternal and neonatal outcomes. The difference in the risk of failed trial of labor may be due to the population of obese women included. Obese women who had a successful ECV may have 
been more motivated during their trial of labor and thus may have been managed differently in labor (e.g., allowed longer duration for labor progress).

There are some limitations that must be acknowledged. Data were extracted from an administrative dataset thus may include data entry errors or miscoding. We excluded incomplete records to minimize such errors. We were also limited to the variables collected; thus, we were not able to control for other potential confounding variables such as amniotic fluid index, anesthesia or tocolytic use, and could not determine how many women were offered an ECV. In a study evaluating the prevalence of ECV among patients with breech presentations, only $10.5 \%$ of patients underwent an ECV while $67.2 \%$ of patients, deemed potentially eligible, did not undergo ECV. In addition, there were more morbidly obese women in the group of eligible women who did not undergo ECV compared with the eligible women that underwent ECV. ${ }^{35}$ Therefore, the obese women available in this analysis may have been motivated to receive an ECV and may have had multiple attempts at ECV, leading to a higher success rate. However, among the women who underwent an attempt, we do not expect a systematic difference in the way an ECV was performed for an obese patient with a prior cesarean delivery compared with a normal weight patient with a prior cesarean delivery.

There are several strengths to this study. We were able to evaluate a large number of obese women with ECV attempts $(n=742)$, thus allowing us to confirm efficacy of ECV among obese women. In addition, we were able to evaluate the risk of adverse maternal and neonatal outcomes between women with an ECV attempt and women with a repeat cesarean delivery, without a trial of labor, with fetal breech presentation among obese women. Thus, when counseling patients with fetal breech presentation and a prior cesarean delivery, providers can use this information.

In conclusion, maternal obesity was not correlated with the success rate of ECV among women with one prior cesarean delivery. In addition, maternal obesity had no effect on the rates of vaginal birth after cesarean delivery. The rates of adverse maternal and neonatal outcomes were similar between obese women who had an attempted ECV and obese women who had a repeat cesarean delivery for breech presentation. Given the similar success rates and adverse outcomes, obese women should be offered ECV.

\section{Note}

This study was presented as a poster presentation at SMFM 40th Annual Meeting, The Pregnancy Meeting; Grapevine, TX on February 6, 2020.

\section{Conflict of Interest}

None declared.

\section{References}

1 Martin JA, Hamilton BE, Osterman MJK. Births in the United States, 2018. NCHS Data Brief 2019;(346):1-8
2 World Health Organization Human Reproduction Programme, 10 April 2015. WHO statement on caesarean section rates. Reprod Health Matters 2015;23(45):149-150

3 Molina G, Weiser TG, Lipsitz SR, et al. Relationship between cesarean delivery rate and maternal and neonatal mortality. JAMA 2015;314(21):2263-2270

4 American College of Obstetricians and Gynecologists. Safe prevention of the primary cesarean delivery. Obstetric care consensus no. 1. . Obstet Gynecol 2014;123:693-711

5 American College of Obstetricians and Gynecologists. External cephalic version. practice bulletin no. 161. Obstet Gynecol 2016; 127:e54-e61

6 American College of Obstetricians and Gynecologists. Vaginal birth after cesarean delivery. ACOG practice bulletin no. 205. Obstet Gynecol 2019;133:e110-e127

7 Attanasio LB, Kozhimannil KB, Kjerulff KH. Women's preference for vaginal birth after a first delivery by cesarean. Birth 2019;46 (01):51-60

8 Hamilton BE, Martin JA, Osterman MJK, Rossen LM. Births: Provisional data for 2018. Vital Statistics Rapid Release; no 7. Hyattsville, MD: National Center for Health Statistics; 2019

9 Morton R, Burton AE, Kumar P, et al. Cesarean delivery: trend in indications over three decades within a major city hospital network. Acta Obstet Gynecol Scand 2020

10 Brocks V, Philipsen T, Secher NJ. A randomized trial of external cephalic version with tocolysis in late pregnancy. $\mathrm{Br} \mathrm{J}$ Obstet Gynaecol 1984;91(07):653-656

11 Mahomed K, Seeras R, Coulson R. External cephalic version at term. A randomized controlled trial using tocolysis. $\mathrm{Br} \mathrm{J}$ Obstet Gynaecol 1991;98(01):8-13

12 Flamm BL, Fried MW, Lonky NM, Giles WS. External cephalic version after previous cesarean section. Am J Obstet Gynecol 1991;165(02):370-372

13 Impey ORE, Greenwood CEL, Impey LWM. External cephalic version after previous cesarean section: a cohort study of 100 consecutive attempts. Eur J Obstet Gynecol Reprod Biol 2018; 231:210-213

14 Schachter M, Kogan S, Blickstein I. External cephalic version after previous cesarean section-a clinical dilemma. Int J Gynaecol Obstet 1994;45(01):17-20

15 de Meeus JB, Ellia F, Magnin G. External cephalic version after previous cesarean section: a series of 38 cases. Eur J Obstet Gynecol Reprod Biol 1998;81(01):65-68

16 Abenhaim HA, Varin J, Boucher M. External cephalic version among women with a previous cesarean delivery: report on 36 cases and review of the literature. J Perinat Med 2009;37(02): $156-160$

17 Weill Y, Pollack RN. The efficacy and safety of external cephalic version after a previous caesarean delivery. Aust N Z J Obstet Gynaecol 2017;57(03):323-326

18 Sela HY, Fiegenberg T, Ben-Meir A, Elchalal U, Ezra Y. Safety and efficacy of external cephalic version for women with a previous cesarean delivery. Eur J Obstet Gynecol Reprod Biol 2009;142(02): 111-114

19 Regalia AL, Curiel P, Natale N, et al. Routine use of external cephalic version in three hospitals. Birth 2000;27(01):19-24

20 Keepanasseril A, Anand K, Soundara Raghavan S. Matched cohort study of external cephalic version in women with previous cesarean delivery. Int J Gynaecol Obstet 2017;138(01):79-83

21 McLaren RA Jr, Atallah F, Fisher N, Minkoff H. Maternal and neonatal outcomes after attempted external cephalic version among women with one previous cesarean delivery. AJP Rep 2018;8(04):e349-e354

22 American College of Obstetricians and Gynecologists Obesity in pregnancy. Practice bulletin no. 156. Obstet Gynecol 2015;126: e112-e126 
23 Behavioral Risk Factor Surveillance System, Centers for Disease Control and Prevention. Available at: www.marchofdimes.org/ peristats. Accessed February 24, 2020

24 Chaudhary S, Contag S, Yao R. The impact of maternal body mass index on external cephalic version success. J Matern Fetal Neonatal Med 2019;32(13):2159-2165

25 Datta S, Cloke B, Harding K, Treharne I. What is the impact of body mass index on external cephalic version. BJOG 2014;121 (03):374

26 Ben-Meir A, Erez Y, Sela HY, Shveiky D, Tsafrir A, Ezra Y. Prognostic parameters for successful external cephalic version.J Matern Fetal Neonatal Med 2008;21(09):660-662

27 Mauldin JG, Mauldin PD, Feng TI, Adams EK, Durkalski VL. Determining the clinical efficacy and cost savings of successful external cephalic version. Am J Obstet Gynecol 1996;175(06): 1639-1644

28 Hellström AC, Nilsson B, Stånge L, Nylund L. When does external cephalic version succeed? Acta Obstet Gynecol Scand 1990;69 (04):281-285

29 Shalev E, Battino S, Giladi Y, Edelstein S. External cephalic version at term-using tocolysis. Acta Obstet Gynecol Scand 1993;72(06): $455-457$
30 Newman RB, Peacock BS, VanDorsten JP, Hunt HH. Predicting success of external cephalic version. Am J Obstet Gynecol 1993; 169(2 Pt 1):245-249, discussion 249-250

31 Tan GW, Jen SW, Tan SL, Salmon YM. A prospective randomised controlled trial of external cephalic version comparing two methods of uterine tocolysis with a non-tocolysis group. Singapore Med J 1989;30(02):155-158

32 Yao R, Crimmins SD, Contag SA, Kopelman JN, Goetzinger KR. Adverse perinatal outcomes associated with trial of labor after cesarean section at term in pregnancies complicated by maternal obesity. J Matern Fetal Neonatal Med 2019;32(08):1256-1261

33 Grasch JL, Thompson JL, Newton JM, Zhai AW, Osmundson SS. Trial of labor compared with cesarean delivery in superobese women. Obstet Gynecol 2017;130(05):994-1000

34 Hibbard JU, Gilbert S, Landon MB, et al; National Institute of Child Health and Human Development Maternal-Fetal Medicine Units Network. Trial of labor or repeat cesarean delivery in women with morbid obesity and previous cesarean delivery. Obstet Gynecol 2006;108(01):125-133

35 Bin YS, Roberts CL, Nicholl MC, Ford JB. Uptake of external cephalic version for term breech presentation: an Australian population study, 2002-2012. BMC Pregnancy Childbirth 2017;17(01):244 